\title{
LA PRESIÓN FISCAL EN LAS COOPERATIVAS: UNA VALORACIÓN POR TAMAÑOS, COMUNIDADES Y SECTORES PARA EL PERÍODO 2008-2011
}

\author{
POR \\ Julián MARTÍNEZ VARGAS ${ }^{2}$, \\ Pedro CARMONA IBÁÑEZ ${ }^{3}$ y \\ José POZUELO CAMPILLO ${ }^{4}$
}

\section{RESUMEN}

Las cooperativas son sociedades con unas características especiales tanto en su regulación como en su tributación, tradicionalmente más ventajosa, a las que se les atribuye importantes funciones sociales.

En este trabajo realizamos un cálculo del impuesto sobre sociedades pagado con objeto de medir la presión fiscal de las empresas cooperativas y de esta forma valorar si se producen diferencias estadísticamente significativas entre los diferentes tipos de estas empresas, según tamaños, sectores y comunidades autónomas.

Para ello partimos de la información adicional de tipo fiscal que ofrecen los balances anuales a partir del año 2008, como consecuencia de la adaptación de la legislación mercantil a las Normas Internacionales de Contabilidad y trabajamos sobre una muestra de empresas cooperativas durante cuatro años.

Palabras clave: cooperativas, fiscalidad, tipo impositivo efectivo, presión fiscal.

\footnotetext{
${ }^{1}$ Trabajo realizado gracias a la financiación de la Generalitat Valenciana para la realización de proyectos de I+D para grupos de investigación emergentes mediante el Proyecto GC/2012/052 correspondiente al ejercicio 2012. Agradecemos a los revisores sus sugerencias y comentarios que sin duda han contribuido a mejorar el texto definitivo.

${ }^{2}$ Profesor Titular. Departamento de Contabilidad. Facultad de Economía. Universidad de Valencia. Dirección de correo electrónico: julian.martinez@uv.es.

${ }^{3}$ Profesor Titular. Departamento de Contabilidad. Facultad de Economía. Universidad de Valencia. Dirección de correo electrónico: pedro.carmona@uv.es.

${ }^{4}$ Profesor Titular. Departamento de Contabilidad. Facultad de Economía. Universidad de Valencia. Dirección de correo electrónico: jose.pozueloz@uv.es.

REVESCO No 119 - Tercer Cuatrimestre 2015 - ISSN: 1885-8031 - www.ucm.es/info/revesco

http://dx.doi.org/10.5209/rev_REVE.2015.n119.49070
}

Fecha de recepción: 02/06/2014

Fecha de aceptación: 26/03/2015 
Claves Econlit: M41; P13; P51.

\title{
FISCAL PRESSURE IN COOPERATIVES: A STUDY BY COMPANY SIZE, REGIONS AND INDUSTRIES FOR THE PERIOD 2008-2011
}

\begin{abstract}
Cooperatives consist of companies with special characteristics both in their regulation and in their taxation, traditionally most advantageous, that have important social functions.

In this paper we obtain the corporate tax paid in order to measure the tax burden of cooperative enterprises and thus determine whether there are statistically significant differences by company size, regions and industries for the period 2008-2011.

To this end we have used the new mandatory fiscal additional information disclosed in the annual financial statement from 2008, after the adaptation of business regulation to the international financial reporting standards in Spain.
\end{abstract}

Keywords: cooperatives, taxation, effective tax rate, tax burden.

\section{INTRODUCCIÓN}

En la mayoría de los trabajos desarrollados en España con el objetivo de valorar la presión fiscal de las sociedades se suele recurrir al impuesto sobre beneficios devengado como aproximación del pago realizado a la Hacienda Pública, aun sabiendo que no tiene por qué coincidir con la cantidad verdaderamente pagada. La justificación de esta práctica la encontramos en la insuficiente información que ofrecen el Balance y la Cuenta de Resultados, estados que habitualmente se utilizan en este tipo de análisis al permitir seleccionar muestras de empresas razonablemente amplias, pues en el caso de querer obtener el importe del impuesto pagado habría que recurrir a datos de la Memoria, no incluidos normalmente en las bases de datos más habituales, lo que merma considerablemente la muestra objeto de estudio.

Sin embargo, con los cambios producidos en las cuentas anuales para su adaptación a la normativa internacional, la información derivada de la contabilización del efecto impositivo adquiere más protagonismo en el Balance de Situación, por lo que se abre la posibilidad de obtener el importe del impuesto sobre beneficios pagado por las empresas, lo que permite medir su presión fiscal. 
Partiendo de esta base y teniendo en cuenta que las cooperativas son entidades jurídicas que tradicionalmente se han tratado de impulsar por su desempeño social, para lo que es fundamental "un buen trato fiscal", aprovechamos la oportunidad que nos da la nueva normativa para valorar la presión fiscal de este tipo de empresas y comprobar si se producen discrepancias importantes como consecuencia de la comunidad autónoma en la que desarrollan su actividad, su tamaño o el sector productivo al que pertenecen.

\section{CONSIDERACIONES FISCALES DE LAS COOPERATIVAS}

Las cooperativas tienen sus normas fiscales específicas aprobadas por la Ley 20/1990 (Régimen fiscal de cooperativas - RFC en adelante) ${ }^{5}$, aunque deben aplicar el régimen general (Texto Refundido de la Ley del Impuesto sobre Sociedades en el período objeto de estudio y Ley 27/2014 del Impuesto sobre Sociedades en la actualidad) en las cuestiones que no estén expresamente previstas en aquellas.

Según el RFC cabe distinguir entre cooperativas protegidas y especialmente protegidas. En estas últimas se observa un mejor trato fiscal al querer incentivar la modernización y desarrollo de sectores económicos como la agricultura, la ganadería y la pesca. Además, se establece una serie de ventajas fiscales que podemos resumir, sin ser exhaustivos, en los siguientes puntos:

1. Tributación de los resultados cooperativos a un tipo del 20\% (los no cooperativos al tipo general).

2. Consideración como gasto deducible del $50 \%$ de la parte de los resultados que se destine obligatoriamente al fondo de reserva obligatorio, partida que se corresponde con la reserva legal en las sociedades capitalistas.

3. Consideración como gasto deducible de la dotación al fondo de educación, formación y promoción aprobado por la asamblea general de la cooperativa, con el límite del $30 \%$ de los excedentes netos del ejercicio.

4. Consideración como gasto deducible de los intereses devengados por las aportaciones de los socios y asociados al capital social con un límite.

5. Una bonificación del $50 \%$ de la cuota íntegra para las cooperativas especialmente protegidas.

\footnotetext{
${ }^{5}$ Esta normativa no es de aplicación en los Territorios Históricos del País Vasco y Navarra que cuentan con sus propias leyes forales.
}

REVESCO No 119 - Tercer Cuatrimestre 2015 - ISSN: 1885-8031 - www.ucm.es/info/revesco 
El tipo impositivo del $20 \%$ puede parecer ventajoso, pero pensamos que lo era más al aprobarse la Ley de RFC en 1990, cuando el tipo impositivo único para el resto de empresas era del $35 \%$. Desde entonces, los tipos del régimen general se han ido reduciendo progresivamente, fijándose un tipo impositivo general a partir de 2016 del 25\%, mientras que el tipo impositivo aplicable a los resultados cooperativos del RFC no ha variado en todo este tiempo. Por tanto, esta ventaja fiscal ha ido perdido interés, sobre todo para las cooperativas pequeñas y medianas que, en ningún caso, han podido acogerse tampoco a los incentivos fiscales establecidos para la empresas de reducida dimensión y ni siquiera aplicar el tipo impositivo reducido a los resultados extracooperativos.

\section{ANTECEDENTES DE ESTUDIOS EMPÍRICOS SOBRE PRESIÓN FISCAL DE} LAS COOPERATIVAS

Cuando se desea medir y valorar la presión fiscal soportada por las empresas en concepto de impuesto sobre beneficios solemos recurrir a una variable denomina tipo impositivo efectivo (TIE), que establece la proporción entre la tributación y el beneficio del cual se ha derivado. El beneficio que ha dado lugar a dicha tributación nos viene proporcionado de forma incuestionable por el resultado contable antes de impuestos. Sin embargo, a la hora de medir la tributación se plantean dos posibilidades; utilizar el gasto por impuesto contabilizado en función del resultado contable o el impuesto realmente pagado en función del resultado fiscal, de tal forma que podemos hablar de un TIE contable, basado en un criterio económico, y un TIE fiscal basado en un criterio fiscal.

TIE Contable $=\frac{\text { Gasto por impuesto sobre sociedades }}{\text { Resultado contable antes de impuestos }}$

TIE Fiscal $=\frac{\text { Cuota líquida del impuesto sobre sociedades }}{\text { Resultado contable antes de impuestos }}$

Como trabajos de referencia que han medido la presión fiscal en las empresas a través del TIE podemos citar, entre otros, los de Stickney y McGee (1982), Zimmerman (1983), Porcano (1986), Wang (1991), Omer et al. (1993), Collins y Shackelford (1995), Gupta y Newberry (1997), Kim y Limpaphayom (1998), Buijink et al. (2002), Fernández (2004), Calvé et al. (2005), Feeny et al. (2005), Richardson y Lanins (2007), Martínez (2006), Fernández y Martínez (2009) y Chen et al. (2009). Sin embargo, teniendo en cuenta las dificultades anteriores al PGC-2007 para obtener el dato de la cuota líquida o impuesto pagado por las empresas, la mayoría de los escasos trabajos que se han hecho en España sobre 
el tipo impositivo efectivo han optado por el TIE contable, desechando los datos de las empresas con un resultado antes de impuestos negativo debido a que un gasto devengado negativo (por contabilización de créditos impositivos) nos devolvería un TIE positivo, lo que supone una verdadera incongruencia. Estos estudios, que tienen como referencia los realizados en otros países y cuyo interés es incuestionable, ya que además de permitir las comparaciones entre países, tratan de relacionar la influencia en la presión fiscal de las empresas de factores tales como el ejercicio, la localización geográfica, la normativa vigente, el sector, la situación económica, la estructura económica y financiera, las inversiones en activos no corrientes, la inversión en $\mathrm{I}+\mathrm{D}+\mathrm{i}$, la estructura financiera, la rentabilidad, etc. Pero casi siempre con la limitación de tener que utilizar un TIE contable por cuestiones prácticas debido a la dificultad de obtener el dato del impuesto pagado a partir de los datos proporcionados por las bases de datos. Tal es así que los trabajos que han apostado por la obtención del impuesto pagado utilizando la información fiscal de la memoria de las propias empresas han sido frecuentemente criticados por la escasez de empresas en la muestra objeto de estudio.

De los trabajos sobre presión fiscal centrados exclusivamente en las cooperativas cabe citar a Molina (2012) que analiza mediante un estudio empírico la presión fiscal (TIE contable) de las cooperativas durante el sexenio 2003-2008, comparando con las sociedades mercantiles y estableciendo una diferencia aproximada de algo más de once puntos porcentuales entre las cooperativas en régimen general y las que cuentan con un régimen fiscal foral como Navarra y el País Vasco, lógicamente a favor de estas últimas. En esta línea, Pozuelo et al. (2012) analizan la estructura económica y financiera de las cooperativas de la Comunidad Valenciana en comparación con las empresas capitalistas en los años 2006 y 2007, deduciendo unos valores en presión fiscal significativamente superiores en estas últimas y estableciendo un TIE contable para las cooperativas entorno al 15,75\% para el período 2006/07.

Otros trabajos como el de Garrido y Puentes (2006) circunscriben el estudio de la presión fiscal a un segmento muy concreto, en este caso las cooperativas agrarias andaluzas de segundo grado, estableciendo que el TIE contable no es representativo de la verdadera presión fiscal debido a que se han cometido errores en la determinación del gasto por impuesto. 
Recientemente, en el trabajo de Alguacil y Romero (2013), se analizan las diferencias territoriales en materia fiscal debidas a las diversas legislaciones autonómicas o la Ley General de Cooperativas, en su caso, observando que el tipo impositivo efectivo derivado de los resultados cooperativos se sitúa en torno al 18\%, con la excepción de Cataluña cuya presión fiscal se reduce al 15\%, como consecuencia de las mayores dotaciones al fondo de reserva obligatorio y al fondo de educación y promoción. En cuanto a los resultados extracooperativos, destaca la diferencia de presión fiscal entre Andalucía $(14,6 \%)$, por un lado y Navarra y Castilla-La Mancha (27\%), cuyas dotaciones a estos mismos fondos es menos exigente en sus correspondientes leyes sustantivas.

Si tomamos como referencia los datos macroeconómicos aportados por la Agencia Tributaria según recaudación del impuesto sobre sociedades en las cooperativas (Tabla 1), podemos observar como el TIE estuvo entorno al $14 \%$ hasta el año 2007 , cayendo al $11,5 \%$ en los años posteriores.

Tabla 1. Cálculo TIE fiscal por años (En miles de euros)

\begin{tabular}{|l|r|r|r|r|r|r|}
\hline & \multicolumn{1}{|c|}{$\mathbf{2 0 0 5}$} & \multicolumn{1}{c|}{$\mathbf{2 0 0 6}$} & \multicolumn{1}{c|}{$\mathbf{2 0 0 7}$} & \multicolumn{1}{c|}{$\mathbf{2 0 0 8}$} & \multicolumn{1}{c|}{$\mathbf{2 0 0 9}$} & \multicolumn{1}{c|}{$\mathbf{2 0 1 0}$} \\
\hline BASE IMPONIBLE & 815.506 & 868.801 & 764.379 & 639.670 & 481.460 & 504.011 \\
\hline CUOTA LÍQUIDA > 0 & 117.282 & 125.733 & 106.868 & 73.726 & 57.133 & 57.457 \\
\hline TIE FISCAL & 0,1438 & 0,1447 & 0,1398 & 0,1153 & 0,1187 & 0,1140 \\
\hline
\end{tabular}

Fuente: Elaboración propia a partir de las estadísticas tributarias de la Agencia Tributaria

Consideramos que la bajada de la presión fiscal podría estar justificada en parte por la reducción en 5 puntos porcentuales del tipo impositivo general que tuvo lugar entre 2007 y 2008, lo que también afectará a las cooperativas en la tributación de sus resultados extracooperativos, pero también coincide con el inicio de la crisis económica con lo que puede haber incidido de alguna forma.

Con los resultados del estudio empírico que realizamos trataremos de contrastar todos estos datos poniendo de manifiesto las principales coincidencias y divergencias. 


\section{CÁLCULO DEL IMPUESTO PAGADO POR LAS COOPERATIVAS}

Para la obtención del impuesto pagado vamos a seguir la formulación propuesta por Martínez (2014) ${ }^{6}$, según la cual utilizaremos los siguientes datos del Balance y la Cuenta de resultados:

\section{$\underline{\text { ACTIVO }}$}

\section{A) ACTIVO NO CORRIENTE}

VI. Activos por impuesto diferido (AID)

\section{PATRIMONIO NETO Y PASIVO}

\section{A) PATRIMONIO NETO}

A-2) Ajustes por cambio de valor (ACV)

A-3) Subvenciones, donaciones y legados recibidos (SDL)

\section{B) PASIVO NO CORRIENTE}

IV. Pasivos por impuesto diferido (PID)

\section{CUENTA DE PÉRDIDAS Y GANANCIAS}

17. Impuesto sobre beneficios (ID)

De tal forma que el impuesto a pagar (IP) los obtendremos de la siguiente forma:

$$
\mathrm{IP}=\mathrm{ID}+\Delta \mathrm{AID}-\Delta \mathrm{PID}+\frac{\mathrm{T}}{1-\mathrm{T}}(\Delta \mathrm{ACV}+\Delta \mathrm{SDL})
$$

El problema está en el tipo impositivo (T) a utilizar en el caso de las cooperativas, ya que puede variar del tipo general del $30 \%$ para los resultados extracooperativos, al 20\% para los cooperativos de las protegidas o el 10\% (por la bonificación del 50\% de la cuota íntegra) en las especialmente protegidas. Partiendo de la base de que un número muy importante de las cooperativas van a ser especialmente protegidas y que en el caso de las pequeñas y medianas

\footnotetext{
${ }^{6}$ En este trabajo podemos ver una justificación detallada de cómo, a partir de los datos del balance y de la cuenta de resultados se puede obtener el impuesto pagado por las empresas, además de valorar las diferentes formas de obtener el tipo impositivo efectivo.
} 
esta parte de la formulación va a tener poca repercusión en el cálculo del impuesto a pagar por ser muy escasas sus variaciones en el patrimonio neto, vamos a optar por la utilización de un tipo impositivo en la formulación del 10\%. No obstante, debemos tener en cuenta que en la formulación propuesta, la principal diferencia del impuesto pagado con el impuesto devengado va a estar en los activos y pasivos por impuesto diferido, cuyo efecto impositivo ya debe tener en cuenta el tipo impositivo que le corresponda a cada cooperativa en la valoración de esos activos y pasivos fiscales. De esta forma, el cálculo del impuesto pagado lo realizaremos con los siguientes cálculos:

$$
\text { IMPUESTO PAGADO }(\mathrm{IP})=\mathrm{ID}+\Delta \mathrm{AID}-\Delta \mathrm{PID}+0,1111(\Delta \mathrm{ACV}+\Delta \mathrm{SDL})
$$

El impuesto pagado obtenido será una cantidad positiva o nula, pudiendo darse el caso de obtener una cantidad negativa en aquellos casos en los que la empresa pertenezca a un grupo que tribute de forma consolidada y éste haya aprovechado su base imponible negativa o deducciones, que individualmente no podría haber compensado, contabilizando en ese caso un ingreso por impuesto corriente equivalente a su efecto impositivo. En este último caso consideraremos simplemente que no se han pagado impuestos. Para contrastar las cantidades obtenidas como pago del impuesto, hemos recurrido a la información fiscal adicional proporcionada por la memoria de algunas de las empresas de la muestra y hemos podido comprobar que el grado de acierto ha estado entre el 90 y el $100 \%$, por lo que consideramos que es una buena aproximación.

Además, como media del período analizado (2008-11) consideraremos los datos agregados de todos los años, utilizando también los de 2007 para obtener las variaciones. Por tanto, cada observación está compuesta en realidad por los datos agregados de cuatro años. Es decir, el tipo impositivo efectivo se ha calculado como el cociente de la suma de los impuestos pagados (según cálculo) en los años 2008-2011 y la suma del resultado antes de impuestos de estos años.

\section{SELECCIÓN Y FUENTES DE LA MUESTRA DE EMPRESAS}

En el proceso de selección y obtención de las diferentes muestras de empresas se ha recurrido a la base de datos financieros $S A B I$ (Sistema de Análisis de Balances Ibéricos), cuyo uso ha sido cedido a la Universidad de Valencia por la empresa INFORMA S.A. 
Para seleccionar las empresas que formarán parte de la muestra de estudio se han considerado solamente empresas cooperativas españolas obteniendo en principio un total de 2.426 distribuidas en diferentes tamaños. Se ha asumido como criterio cuantitativo de tamaño el número de trabajadores que conforman la plantilla de las empresas consideradas, mostrados en la Tabla 2:

Tabla 2. Criterios cuantitativos para la clasificación de empresas

\begin{tabular}{|c|c|c|c|c|}
\hline CRITERIOS & $\begin{array}{c}\text { EMPRESA } \\
\text { GRANDE }\end{array}$ & $\begin{array}{c}\text { EMPRESA } \\
\text { MEDIANA }\end{array}$ & $\begin{array}{c}\text { EMPRESA } \\
\text { PEQUEÑA }\end{array}$ & $\begin{array}{c}\text { MICRO } \\
\text { EMPRESA }\end{array}$ \\
\hline $\begin{array}{l}\text { PLANTILLA DE } \\
\text { LA EMPRESA }\end{array}$ & $>250$ & $<250$ & $<50$ & $<10$ \\
\hline
\end{tabular}

Fuente: Comisión de las Comunidades Europeas

Sin embargo, después de realizar una serie de ajustes consistentes en descartar aquellas empresas con observaciones incompletas en cualquiera de los años considerados o valores extremos, la muestra final ha quedado reducida a un número total válido de 1.407 observaciones. La Tabla 3 recoge la distribución de las empresas cooperativas por tamaño.

Tabla 3. Distribución por tamaños de las empresas cooperativas

\begin{tabular}{|l|c|}
\hline \multicolumn{1}{|c|}{ TAMAÑO } & TOTALES \\
\hline GRANDES & 37 \\
\hline MEDIANAS & 84 \\
\hline PEQUEÑAS & 296 \\
\hline MICROEMPRESAS & 990 \\
\hline TOTAL & $\mathbf{1 . 4 0 7}$ \\
\hline
\end{tabular}

Fuente: SABI

En la Tabla 4 se presenta el resumen de la distribución de la muestra de empresas por sectores de actividad económica mostrando el peso específico de cada grupo sectorial en el total de la muestra. La agrupación por sectores se ha realizado atendiendo al C.N.A.E. de 2009 considerando los dos primeros dígitos de control, lo que ha permitido distinguir 14 actividades básicas.

Tabla 4. Distribución de la muestra de empresas por sectores de actividad económica

\begin{tabular}{|c|l|c|}
\hline $\begin{array}{c}\text { CNAE- } \\
\text { 2009 }\end{array}$ & \multicolumn{1}{|c|}{ ACTIVIDAD } & $\begin{array}{c}\mathbf{N}^{\mathbf{0}} \\
\text { EMPRESAS }\end{array}$ \\
\hline $01-03$ & Agricultura, ganadería, selvicultura y pesca & 365 \\
\hline $05-09$ & Industrias extractivas & 2 \\
\hline $10-33$ & Industria manufacturera & 252 \\
\hline 35 & Suministro de energía & 4 \\
\hline
\end{tabular}

REVESCO No 119 - Tercer Cuatrimestre 2015 - ISSN: 1885-8031 - www.ucm.es/info/revesco 


\begin{tabular}{|c|l|c|}
\hline $36-39$ & Suministro de agua & 14 \\
\hline $41-43$ & Construcción & 118 \\
\hline $45-47$ & Comercio al por mayor y al por menor & 404 \\
\hline $49-53$ & Transportes & 63 \\
\hline $55-56$ & Hostelería & 14 \\
\hline $58-63$ & Información y comunicaciones & 12 \\
\hline $64-66$ & Actividades financieras y seguros & 7 \\
\hline $68-75$ & Actividades inmobiliarias & 53 \\
\hline $77-82$ & Actividades administrativas y servicios aux. & 32 \\
\hline & Resto & 67 \\
\hline TOTAL & & $\mathbf{1 . 4 0 7}$ \\
\hline
\end{tabular}

Fuente: Elaboración propia

Por último, en la Tabla 5 se presenta el resumen de la distribución de la muestra de empresas por comunidades autónomas.

Tabla 5. Distribución de la muestra de empresas por CC.AA

\begin{tabular}{|l|c|}
\hline \multicolumn{1}{|c|}{ COMUNIDAD } & N $^{\mathbf{0}}$ EMPRESAS \\
\hline Andalucía & 128 \\
\hline Aragón & 168 \\
\hline Asturias & 49 \\
\hline Baleares & 3 \\
\hline Canarias (*) & 27 \\
\hline Castilla y León & 86 \\
\hline Castilla-La Mancha & 144 \\
\hline Cataluña & 43 \\
\hline Comunidad Valenciana & 312 \\
\hline Extremadura & 219 \\
\hline Galicia & 34 \\
\hline La Rioja & 11 \\
\hline Madrid & 23 \\
\hline Melilla $\left(^{*}\right)$ & 9 \\
\hline Murcia & 35 \\
\hline Navarra & 14 \\
\hline País Vasco & 102 \\
\hline & $\mathbf{1 . 4 0 7}$ \\
\hline
\end{tabular}

Fuente: Elaboración propia.

(*) Comunidades sin ley de cooperativas propia.

A continuación aplicaremos a esta muestra las diferentes técnicas estadísticas que nos permitan alcanzar los objetivos propuestos. 


\section{RESULTADOS}

\subsection{Análisis del tipo impositivo de las sociedades cooperativas por tamaño}

Las sociedades cooperativas se han agrupado de acuerdo con su tamaño, obteniendo de este modo cuatro categorías: gran empresa, mediana, pequeña y micro empresa. La Tabla 6 recoge el resumen de los estadísticos descriptivos del tipo impositivo de acuerdo con este criterio de agrupación. Como primer dato relevante obtenemos un tipo efectivo fiscal medio del 14,78\%, superior al 11,6\% que se desprende de los datos fiscales aportados por la Agencia Tributaria (Tabla 1) en el trienio 2008-10. En cuanto al tamaño, apreciamos una mayor tributación en las cooperativas de tamaño medio, entorno al 17\%, teniendo como referencia mas baja las microempresas con una tributación cercana al 13\%. En este sentido, no coincidimos con Molina (2012) que con un TIE contable establece una relación directa entre tamaño y tipo efectivo, atribuyendo una mayor presión fiscal a las grandes empresas. Según nuestro estudio, al igual que ocurre con las empresas consideradas capitalistas, las grandes cooperativas tienen una tributación efectiva inferior al resto, probablemente debido a la aplicación mayoritaria del régimen de consolidación fiscal por formar parte de un grupo y a la realización de más inversiones en actividades que dan derecho a deducciones (I+D+i, mecenazgo, medioambientales, etc.). En cuanto a las microempresas, consideramos que la menor presión fiscal está justificada por ser más proclives a estar fiscalmente protegidas, con los consiguientes beneficios fiscales, además de que sus resultados extracooperativos, que son los que soportan una mayor tributación, tendrán un menor peso específico en este tipo de empresas.

Tabla 6. Resumen de los estadísticos descriptivos del TIE por tamaño

\begin{tabular}{lrrrrrr}
\hline & Obs. & Media & D. Estándar & Mediana & Mín. & Máx. \\
\hline Gran empresa & 37 & 13,83 & 12,36 & 11,39 & 0 & 40,72 \\
Mediana & 84 & 17,07 & 12,09 & 14,98 & 0 & 50,11 \\
Pequeña & 296 & 15,28 & 12,84 & 14,07 & 0 & 50,72 \\
Micro-empresa & 990 & 12,94 & 13,45 & 9,58 & 0 & 50,76 \\
\hline
\end{tabular}

El Gráfico 1 muestra la representación de estas variables en forma de diagramas de caja. Se aprecia claramente que los menores tipos impositivos corresponden a la gran empresa y la microempresa. En los diagramas de caja también se aprecia que la dispersión de la variable tipo impositivo dentro de los valores centrales entre el primer y tercer cuartil es similar dentro de cada categoría de empresa, salvo para la micro-empresa que es mayor.

REVESCO N 119 - Tercer Cuatrimestre 2015 - ISSN: 1885-8031 - www.ucm.es/info/revesco 
Gráfico 1. Diagrama de caja del tipo impositivo efectivo por tamaño

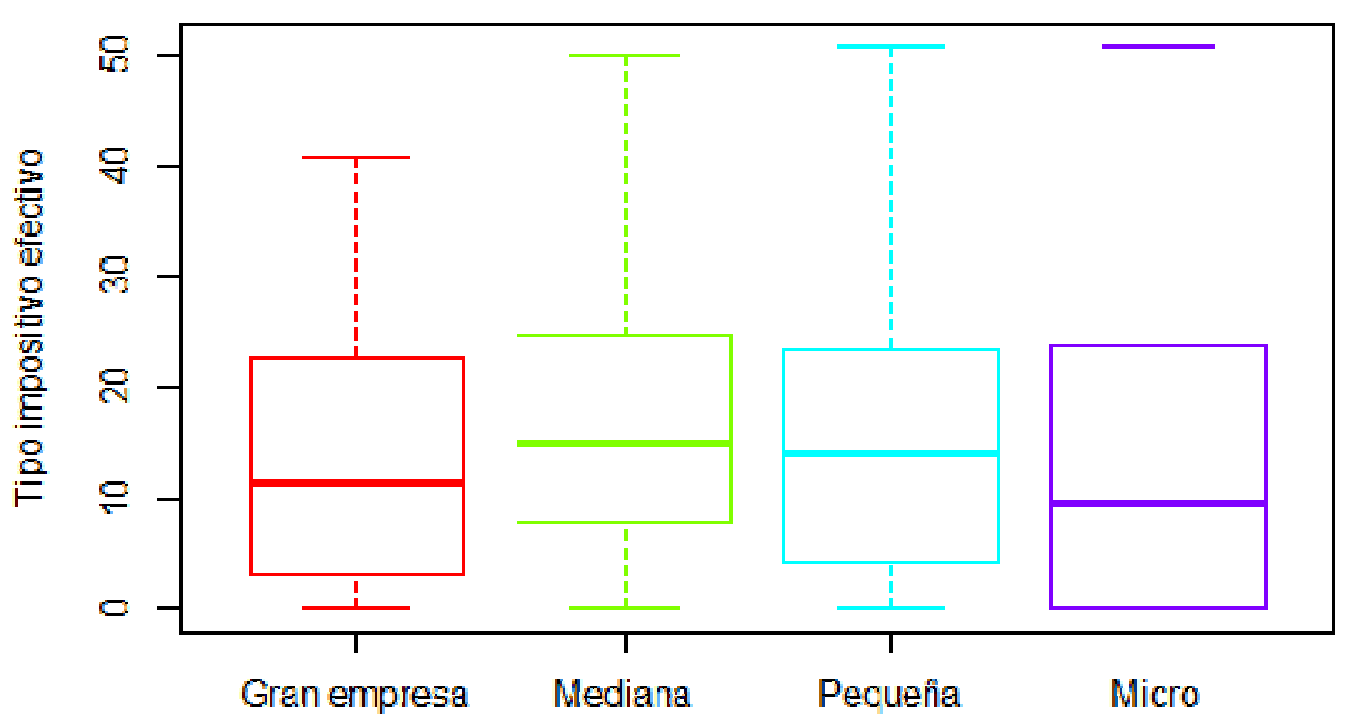

Con el fin de asegurarnos que el tipo impositivo medio es mayor efectivamente para las empresas grandes y para las micro-empresas hemos llevado a cabo una prueba de análisis de varianza de un factor (ANOVA). De acuerdo con el nivel de significación obtenido (véase Tabla 7) podemos afirmar que el nivel impositivo es diferente para los cuatro grupos de sociedades cooperativas, siendo mayor para las empresas grandes y micro-empresas, tal y como se ha reflejado en el análisis descriptivo.

Tabla 7. Prueba univariante del efecto del tamaño en el TIE

\begin{tabular}{crrc}
\hline Variable & $\begin{array}{c}\text { Medias } \\
\text { cuadradas }\end{array}$ & $\boldsymbol{F}$ & Significat. \\
\hline Tamaño & 2263 & 4,315 & 0,0048 \\
\hline
\end{tabular}

También hemos realizado comparaciones múltiples por parejas de las variables que categorizan el tamaño de la empresa con el fin de identificar diferencias estadísticamente significativas. Para tal efecto hemos calculado un conjunto de intervalos de confianza sobre las diferencias de las medias de los distintos niveles del factor tamaño-empresa mediante el método HSD de Tuckey ${ }^{7}$ (Tabla 8). De acuerdo con los resultados obtenidos las

\footnotetext{
${ }^{7}$ Tukey's Honestly Significant Test Differences. Esta prueba estadística compara todos los posibles pares de las medias de cada grupo, de modo que identifica cualquier diferencia entre dos medias que sea mayor que el error estándar esperado

(http://en.wikipedia.org/wiki/Tukey\%27s_range test, consulado el 25/07/13)
} 
comparaciones Micro-Mediana y Micro-Pequeña resultan estadísticamente significativas a un nivel del 5,00\%, siendo el tipo impositivo efectivo inferior en las microempresas.

Por lo tanto, parece evidente que dado que el RFC no ha cambiado en los últimos años, las cooperativas que más se han visto beneficiadas por las mejoras fiscales han sido aquellas que han podido acogerse a los incentivos fiscales de la especial protección del RFC y las deducciones por la realización de determinadas actividades establecidas en el régimen general que suelen aprovechar mejor las grandes empresas.

Tabla 8. Comparaciones múltiples por parejas del tamaño en el TIE

\begin{tabular}{lrrrc}
\hline \multicolumn{1}{c}{ Comparación } & \multicolumn{5}{c}{ Liferite } & Inferior & Superior & P ajust. \\
\hline Mediana-Gran empresa & 3,237 & $-3,474$ & 9,948 & 0,601 \\
Pequeña-Gran empresa & 1,447 & $-4,483$ & 7,378 & 0,923 \\
Micro-Gran empresa & $-0,891$ & $-6,586$ & 4,804 & 0,978 \\
Pequeña-Mediana & $-1,790$ & $-5,994$ & 2,415 & 0,693 \\
Micro-Mediana & $-4,128$ & $-7,993$ & $-0,263$ & 0,031 \\
Micro-Pequeña & $-2,339$ & $-4,592$ & $-0,086$ & $0,038 * *$ \\
\hline
\end{tabular}

NOTAS: límites del intervalo de confianza calculados para un nivel de significatividad del 5\%.

(***) Significativo al nivel del 1,00\%

(**) Significativo al nivel del 5,00\%

(*) Significativo al nivel del 10,00\%

\subsection{Análisis del tipo impositivo de las sociedades cooperativas por regiones}

Con el fin de identificar alguna posible asimetría de carácter regional se ha analizado la distribución del tipo impositivo efectivo por comunidades autónomas. No se han considerado las comunidades de Baleares y Melilla por disponer de un número de observaciones válidas inferior a la decena. En la Tabla 9 mostramos un resumen de los principales estadísticos descriptivos del tipo impositivo por regiones. Con los diagramas de caja se ha representado gráficamente los valores centrales y la dispersión de la variable tipo impositivo efectivo por regiones (Gráfico 2). Se observa que los menores valores impositivos corresponden al País Vasco, Canarias, Comunidad Valenciana y Asturias. En cambio, las comunidades de Castilla y León, Murcia, Andalucía y Galicia presentan tipos impositivos efectivos mayores, que precisamente en el trabajo de Molina (2012) se establecen también como las de máxima tributación, junto con la Rioja (con pocas observaciones), aunque nosotros consideramos que el porcentaje no ha sido superior en ningún caso al $19 \%$ en el período 2008-11. 
Coincidimos también en la inferior tributación de las cooperativas del País Vasco, pero no compartimos esa misma apreciación para las de Navarra, si bien es cierto que el número de observaciones para esta comunidad es de los más reducidos, por lo que las valoraciones tienen un menor peso específico. 
Gráfico 2. Diagrama de caja del tipo impositivo efectivo por comunidad autónoma

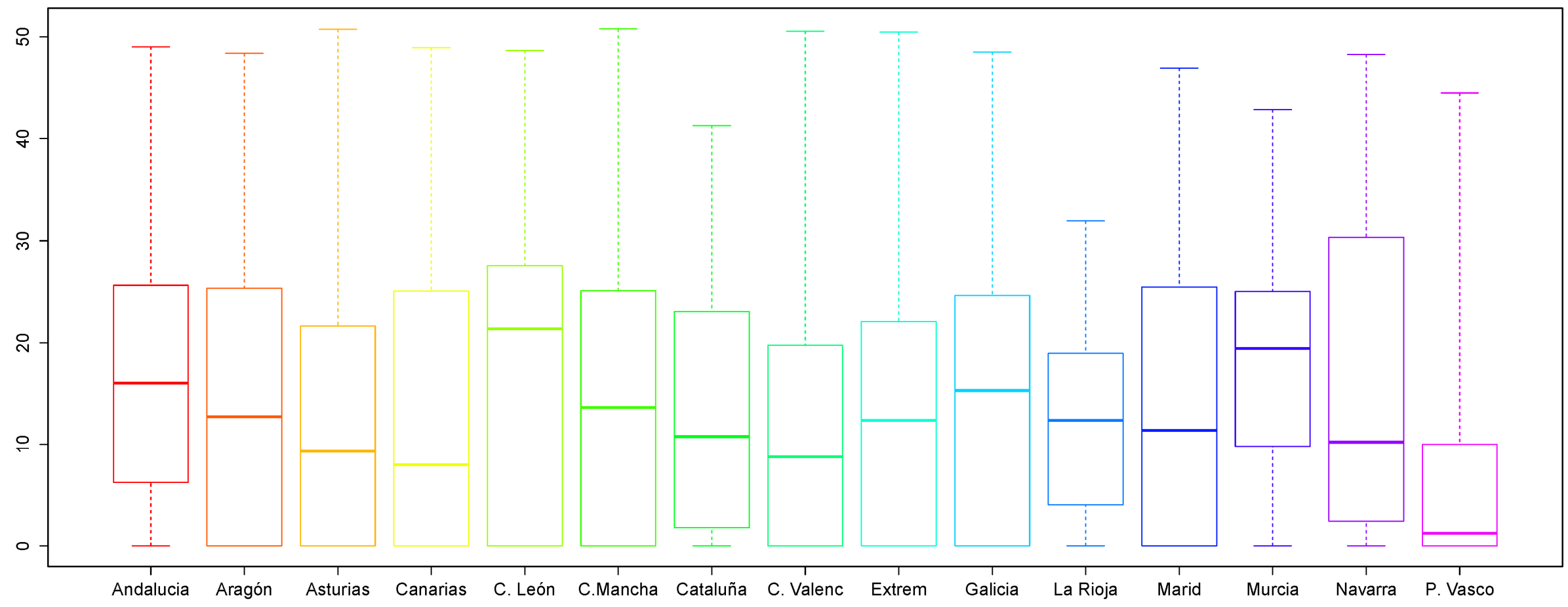


Tabla 9. Resumen de los estadísticos descriptivos del TIE por comunidad autónoma

\begin{tabular}{lrrrrrr}
\hline & Obs, & Media & D. Estándar & Mediana & Mín, & Máx, \\
\hline Andalucia & 128 & 16,65 & 13,35 & 16,01 & 0 & 49,01 \\
Aragón & 168 & 15,53 & 13,58 & 12,72 & 0 & 48,37 \\
Asturias & 49 & 12,44 & 13,90 & 9,30 & 0 & 50,72 \\
Canarias & 27 & 12,84 & 14,13 & 7,98 & 0 & 48,93 \\
C. y León & 86 & 18,21 & 14,64 & 21,36 & 0 & 48,66 \\
C.Mancha & 144 & 15,80 & 14,18 & 13,60 & 0 & 50,76 \\
Cataluña & 43 & 13,40 & 11,31 & 10,77 & 0 & 41,27 \\
C. Valenc & 312 & 11,41 & 12,36 & 8,745 & 0 & 50,55 \\
Extrem & 219 & 14,08 & 13,37 & 12,36 & 0 & 50,45 \\
Galicia & 34 & 14,62 & 12,76 & 15,28 & 0 & 48,51 \\
La Rioja & 11 & 13,24 & 10,61 & 12,37 & 0 & 31,93 \\
Madrid & 23 & 14,12 & 13,60 & 11,39 & 0 & 46,94 \\
Murcia & 35 & 17,71 & 10,83 & 19,45 & 0 & 42,82 \\
Navarra & 14 & 16,58 & 17,02 & 10,22 & 0 & 48,27 \\
P. Vasco & 102 & 6,45 & 9,25 & 1,29 & 0 & 44,45 \\
\hline & & & & & &
\end{tabular}

La Tabla 10 muestra el resultado de la prueba univariante del tipo impositivo efectivo por comunidad autónoma, apreciándose que el nivel de significatividad es muy bajo, lo que supone diferencias estadísticamente significativas entre las distintas comunidades autónomas para el tipo impositivo, tal y como a priori se desprendía del análisis descriptivo.

Tabla 10. Prueba univariante del efecto de la comunidad autónoma en el TIE

\begin{tabular}{crrc}
\hline Variable & $\begin{array}{c}\text { Medias } \\
\text { cuadradas }\end{array}$ & $\boldsymbol{F}$ & Significat. \\
\hline Región & 849,50 & 5,015 & 0,000 \\
\hline
\end{tabular}

Las comparaciones múltiples por parejas del factor o tratamiento pertenencia a una comunidad autónoma para la variable dependiente tipo impositivo efectivo se recoge en la Tabla 11. Algunas de las comparaciones son estadísticamente significativas y el cero no se encuentra entre el límite inferior y superior de los intervalos de confianza, es decir, para algunas comunidades el impuesto es mayor en comparación con otras. 
Tabla 11. Comparaciones múltiples por parejas de la comunidad autónoma en el TIE

\begin{tabular}{|c|c|c|c|c|c|}
\hline \multirow[b]{2}{*}{ Comparación } & \multicolumn{4}{|c|}{ Límite intervalo } & \\
\hline & Difer, & Inferior & Superior & $P$ ajust, & \\
\hline Aragón-Andalucia & $-1,12$ & $-6,31$ & 4,07 & 1,00 & \\
\hline Asturias-Andalucia & $-4,21$ & $-11,64$ & 3,22 & 0,84 & \\
\hline Canarias-Andalucia & $-3,81$ & $-13,17$ & 5,56 & 0,99 & \\
\hline C. León-Andalucia & 1,56 & $-4,60$ & 7,73 & 1,00 & \\
\hline C.Mancha-Andalucia & $-0,85$ & $-6,22$ & 4,52 & 1,00 & \\
\hline Cataluña-Andalucia & $-3,25$ & $-11,04$ & 4,55 & 0,99 & \\
\hline C. Valenc-Andalucia & $-5,24$ & $-9,88$ & $-0,59$ & 0,01 & $* * *$ \\
\hline Extrem-Andalucia & $-2,57$ & $-7,49$ & 2,35 & 0,91 & \\
\hline Galicia-Andalucia & $-2,03$ & $-10,56$ & 6,5 & 1,00 & \\
\hline La Rioja-Andalucia & $-3,41$ & $-17,3$ & 10,48 & 1,00 & \\
\hline Marid-Andalucia & $-2,53$ & $-12,54$ & 7,49 & 1,00 & \\
\hline Murcia-Andalucia & 1,06 & $-7,38$ & 9,49 & 1,00 & \\
\hline Navarra-Andalucia & $-0,07$ & $-12,52$ & 12,38 & 1,00 & \\
\hline P. Vasco-Andalucia & $-10,2$ & $-16,07$ & $-4,33$ & 0,00 & $* * *$ \\
\hline Asturias-Aragón & $-3,1$ & $-10,27$ & 4,08 & 0,98 & \\
\hline Canarias-Aragón & $-2,69$ & $-11,86$ & 6,48 & 1,00 & \\
\hline C. León-Aragón & 2,68 & $-3,18$ & 8,54 & 0,97 & \\
\hline C.Mancha-Aragón & 0,27 & $-4,76$ & 5,29 & 1,00 & \\
\hline Cataluña-Aragón & $-2,13$ & $-9,69$ & 5,43 & 1,00 & \\
\hline C. Valenc-Aragón & $-4,12$ & $-8,35$ & 0,11 & 0,07 & $*$ \\
\hline Extrem-Aragón & $-1,45$ & $-5,99$ & 3,08 & 1,00 & \\
\hline Galicia-Aragón & $-0,91$ & $-9,23$ & 7,41 & 1,00 & \\
\hline La Rioja-Aragón & $-2,29$ & $-16,05$ & 11,47 & 1,00 & \\
\hline Madrid-Aragón & $-1,41$ & $-11,24$ & 8,42 & 1,00 & \\
\hline Murcia-Aragón & 2,17 & $-6,04$ & 10,39 & 1,00 & \\
\hline Navarra-Aragón & 1,05 & $-11,25$ & 13,35 & 1,00 & \\
\hline P. Vasco-Aragón & $-9,08$ & $-14,63$ & $-3,53$ & 0,00 & $* * *$ \\
\hline Canarias-Asturias & 0,41 & $-10,19$ & 11,00 & 1,00 & \\
\hline C. León-Asturias & 5,78 & $-2,14$ & 13,69 & 0,46 & \\
\hline C.Mancha-Asturias & 3,36 & $-3,95$ & 10,67 & 0,97 & \\
\hline Cataluña-Asturias & 0,97 & $-8,27$ & 10,21 & 1,00 & \\
\hline C. Valenc-Asturias & $-1,02$ & $-7,82$ & 5,77 & 1,00 & \\
\hline Extrem-Asturias & 1,64 & $-5,35$ & 8,63 & 1,00 & \\
\hline Galicia-Asturias & 2,19 & $-7,68$ & 12,05 & 1,00 & \\
\hline La Rioja-Asturias & 0,8 & $-13,95$ & 15,56 & 1,00 & \\
\hline Marid-Asturias & 1,68 & $-9,49$ & 12,86 & 1,00 & \\
\hline Murcia-Asturias & 5,27 & $-4,52$ & 15,05 & 0,89 & \\
\hline P. Vasco-Asturias & $-5,99$ & $-13,67$ & 1,70 & 0,34 & \\
\hline C. León-Canarias & 5,37 & $-4,39$ & 15,12 & 0,87 & \\
\hline C.Mancha-Canarias & 2,95 & $-6,32$ & 12,23 & 1,00 & \\
\hline Cataluña-Canarias & 0,56 & $-10,3$ & 11,42 & 1,00 & \\
\hline C. Valenc-Canarias & $-1,43$ & $-10,3$ & 7,44 & 1,00 & \\
\hline Extrem-Canarias & 1,23 & $-7,78$ & 10,25 & 1,00 & \\
\hline Galicia-Canarias & 1,78 & $-9,62$ & 13,18 & 1,00 & \\
\hline La Rioja-Canarias & 0,4 & $-15,42$ & 16,21 & 1,00 & \\
\hline Madrid-Canarias & 1,28 & $-11,27$ & 13,82 & 1,00 & \\
\hline Murcia-Canarias & 4,86 & $-6,46$ & 16,19 & 0,98 & \\
\hline Navarra-Canarias & 3,74 & $-10,83$ & 18,3 & 1,00 & \\
\hline P. Vasco-Canarias & $-6,4$ & $-15,97$ & 3,17 & 0,61 & \\
\hline Navarra-Asturias & 4,14 & $-9,26$ & 17,54 & 1,00 & \\
\hline
\end{tabular}


Tabla 11. Comparaciones múltiples por parejas de la comunidad autónoma en el TIE (Continuación)

\begin{tabular}{|c|c|c|c|c|c|}
\hline \multicolumn{6}{|c|}{ Límite intervalo } \\
\hline Comparación & Difer. & Inferior & Superior & $P$ ajust. & \\
\hline C.Mancha-C. León & $-2,41$ & $-8,44$ & 3,61 & 0,99 & \\
\hline Cataluña-C. León & $-4,81$ & $-13,07$ & 3,45 & 0,81 & \\
\hline C. Valenc-C. León & $-6,8$ & $-12,18$ & $-1,41$ & 0,00 & $* * *$ \\
\hline Extrem-C. León & $-4,13$ & $-9,76$ & 1,49 & 0,44 & \\
\hline Galicia-C. León & $-3,59$ & $-12,55$ & 5,37 & 0,99 & \\
\hline La Rioja-C. León & $-4,97$ & $-19,13$ & 9,19 & 1,00 & \\
\hline Madrid-C. León & $-4,09$ & $-14,47$ & 6,29 & 0,99 & \\
\hline Murcia-C. León & $-0,51$ & $-9,37$ & 8,36 & 1,00 & \\
\hline Navarra-C. León & $-1,63$ & $-14,38$ & 11,11 & 1,00 & \\
\hline P. Vasco-C. León & $-11,77$ & $-18,24$ & $-5,29$ & 0,00 & $* * *$ \\
\hline Cataluña-C.Mancha & $-2,4$ & $-10,08$ & 5,29 & 1,00 & \\
\hline C. Valenc-C.Mancha & $-4,38$ & $-8,84$ & 0,07 & 0,06 & $*$ \\
\hline Extrem-C.Mancha & $-1,72$ & $-6,46$ & 3,02 & 1,00 & \\
\hline Galicia-C.Mancha & $-1,18$ & $-9,61$ & 7,25 & 1,00 & \\
\hline La Rioja-C.Mancha & $-2,56$ & $-16,39$ & 11,27 & 1,00 & \\
\hline Madrid-C.Mancha & $-1,68$ & $-11,61$ & 8,25 & 1,00 & \\
\hline Murcia-C.Mancha & 1,91 & $-6,43$ & 10,24 & 1,00 & \\
\hline Navarra-C.Mancha & 0,78 & $-11,6$ & 13,16 & 1,00 & \\
\hline P. Vasco-C.Mancha & $-9,35$ & $-15,07$ & $-3,63$ & 0,00 & $* * *$ \\
\hline C. Valenc-Cataluña & $-1,99$ & $-9,18$ & 5,20 & 1,00 & \\
\hline Extrem-Cataluña & 0,68 & $-6,7$ & 8,05 & 1,00 & \\
\hline Galicia-Cataluña & 1,22 & $-8,93$ & 11,37 & 1,00 & \\
\hline La Rioja-Cataluña & $-0,16$ & $-15,1$ & 14,78 & 1,00 & \\
\hline Madrid-Cataluña & 0,72 & $-10,7$ & 12,14 & 1,00 & \\
\hline Murcia-Cataluña & 4,3 & $-5,76$ & 14,37 & 0,98 & \\
\hline Navarra-Cataluña & 3,18 & $-10,43$ & 16,78 & 1,00 & \\
\hline P. Vasco-Cataluña & $-6,95$ & $-14,99$ & 1,08 & 0,18 & \\
\hline Extrem-C. Valenc & 2,66 & $-1,23$ & 6,56 & 0,57 & \\
\hline Galicia-C. Valenc & 3,21 & $-4,78$ & 11,19 & 0,99 & \\
\hline La Rioja-C. Valenc & 1,83 & $-11,74$ & 15,39 & 1,00 & \\
\hline Madrid-C. Valenc & 2,71 & $-6,85$ & 12,26 & 1,00 & \\
\hline Murcia-C. Valenc & 6,29 & $-1,59$ & 14,17 & 0,30 & \\
\hline Navarra-C. Valenc & 5,17 & $-6,91$ & 17,25 & 0,98 & \\
\hline P. Vasco-C. Valenc & $-4,97$ & $-10,01$ & 0,08 & 0,06 & $*$ \\
\hline Galicia-Extrem & 0,54 & $-7,61$ & 8,70 & 1,00 & \\
\hline La Rioja-Extrem & $-0,84$ & $-14,5$ & 12,83 & 1,00 & \\
\hline Madrid-Extrem & 0,04 & $-9,65$ & 9,74 & 1,00 & \\
\hline Murcia-Extrem & 3,63 & $-4,42$ & 11,68 & 0,97 & \\
\hline Navarra-Extrem & 2,5 & $-9,69$ & 14,69 & 1,00 & \\
\hline P. Vasco-Extrem & $-7,63$ & $-12,93$ & $-2,33$ & 0,00 & $* * *$ \\
\hline La Rioja-Galicia & $-1,38$ & $-16,72$ & 13,96 & 1,00 & \\
\hline Madrid-Galicia & $-0,50$ & $-12,44$ & 11,44 & 1,00 & \\
\hline Navarra-Galicia & 1,96 & $-12,08$ & 16,00 & 1,00 & \\
\hline P. Vasco-Galicia & $-8,17$ & $-16,93$ & 0,58 & 0,10 & $*$ \\
\hline Madrid-La Rioja & 0,88 & $-15,33$ & 17,09 & 1,00 & \\
\hline Murcia-La Rioja & 4,46 & $-10,82$ & 19,75 & 1,00 & \\
\hline Navarra-La Rioja & 3,34 & $-14,48$ & 21,15 & 1,00 & \\
\hline P. Vasco-La Rioja & $-6,79$ & $-20,83$ & 7,24 & 0,95 & \\
\hline Murcia-Madrid & 3,58 & $-8,29$ & 15,45 & 1,00 & \\
\hline Navarra-Madrid & 2,46 & $-12,53$ & 17,45 & 1,00 & \\
\hline P. Vasco-Madrid & $-7,67$ & $-17,88$ & 2,53 & 0,40 & \\
\hline Navarra-Murcia & $-1,13$ & $-15,11$ & 12,86 & 1,00 & \\
\hline P. Vasco-Murcia & $-11,26$ & $-19,92$ & $-2,60$ & 0,00 & $* * *$ \\
\hline P. Vasco-Navarra & $-10,13$ & $-22,73$ & 2,47 & 0,29 & \\
\hline Murcia-Galicia & 3,08 & $-7,56$ & 13,73 & 1,00 & \\
\hline
\end{tabular}

REVESCO N 119 - Tercer Cuatrimestre 2015 - ISSN: 1885-8031 - www.ucm.es/info/revesco 
(***) Significativo al nivel del $1,00 \%$

(**) Significativo al nivel del $5,00 \%$

(*) Significativo al nivel del $10,00 \%$

El análisis de los intervalos de confianza y niveles de significatividad nos ha permitido identificar que la mayoría de las diferencias se encuentran en las comparaciones donde aparecen las comunidades autónomas del País Vasco y la Comunidad Valenciana. Estos resultados reafirmarían los obtenidos en el análisis descriptivo en el sentido de que el País Vasco y Comunidad Valenciana serían las regiones con un menor tipo impositivo efectivo. Asimismo, en estas comparaciones estadísticamente significativas, Andalucía, Castilla y León, Extremadura, Aragón, Galicia y Murcia son las que en la en el análisis descriptivo presentan unos mayores tipos impositivos efectivos.

La menor tributación efectiva de las cooperativas del País Vasco se podría justificar por los mayores incentivos fiscales de su normativa foral, principalmente un tipo impositivo del $19 \%$ para las pequeñas empresas y la inclusión adicional como especialmente protegidas de las cooperativas de enseñanza y de viviendas.

En cuanto a las cooperativas de la Comunidad Valenciana, la menor presión fiscal la atribuimos al gran protagonismo que tienen en esta comunidad las que hemos denominado micro-cooperativas, que como se ha visto tienen una menor tributación efectiva y al efecto fiscal añadido de la dotación al FRO y al FEP de la totalidad de los resultados extracooperativos.

\subsection{Análisis del tipo impositivo de las sociedades cooperativas por actividad}

Mediante este tercer análisis se ha comprobado la existencia de diferencias entre las sociedades cooperativas considerando como factor de agrupación la actividad que desempeñan. Para este fin se ha tomado el criterio de ordenación de la CNAE del año 2009 con una extensión de dos dígitos, eliminándose los sectores resultantes con un número de observaciones inferior a la decena. El resumen de los estadísticos descriptivos del tipo impositivo efectivo de acuerdo con este criterio de clasificación se muestra en la Tabla 12. Por su parte, el Gráfico 3 recoge la representación de estos grupos en forma de diagramas de caja. Hostelería, administración y servicios, información y comunicación, construcción y transporte y almacenamiento son los sectores de actividad con un menor tipo impositivo efectivo. En cambio, comercio e inmobiliarias y, sobre todo, agricultura, ganadería y pesca soportan los mayores tipos impositivos efectivos. 
Coincidimos con Molina (2012) en que las cooperativas del sector agrícola, ganadero y pesquero son las menos favorecidas fiscalmente, con una tributación efectiva media que en nuestro período objeto de estudio establecemos entorno al $17 \%$, seguidas de las comerciales con un $15 \%$. Por su lado, la hostelería sería el sector más favorecido fiscalmente, con una tributación efectiva media entorno al $6 \%$.

Tabla 12. Resumen de los estadísticos descriptivos del TIE por sector de actividad

\begin{tabular}{lrrrrrr}
\hline & Obs. & Media & D. Estándar & Mediana & Mín. & Máx. \\
\hline Agric. gan. y pes. & 365 & 16,80 & 14,32 & 17,79 & 0 & 50,76 \\
Industr. manufact. & 252 & 12,26 & 13,46 & 8,84 & 0 & 50,72 \\
Sumintro agua & 14 & 11,83 & 13,68 & 6,67 & 0 & 40,35 \\
Construcción & 118 & 9,37 & 11,96 & 4,91 & 0 & 50,55 \\
Comercio & 404 & 14,88 & 12,47 & 13,0 & 0 & 50,11 \\
Transp. y almac. & 63 & 8,95 & 11,24 & 0,00 & 0 & 50,45 \\
Hostelería & 14 & 5,76 & 8,78 & 0,00 & 0 & 25,01 \\
Inform. y comun. & 12 & 8,23 & 10,91 & 3,15 & 0 & 30,02 \\
Inmobiliarias & 53 & 13,09 & 12,50 & 9,71 & 0 & 42,23 \\
Admin. y servicios & 32 & 7,94 & 9,72 & 3,69 & 0 & 29,51 \\
Resto & 67 & 13,24 & 13,53 & 9,58 & 0 & 46,94 \\
\hline
\end{tabular}

Gráfico 3. Diagrama de caja del tipo impositivo efectivo por sector de actividad

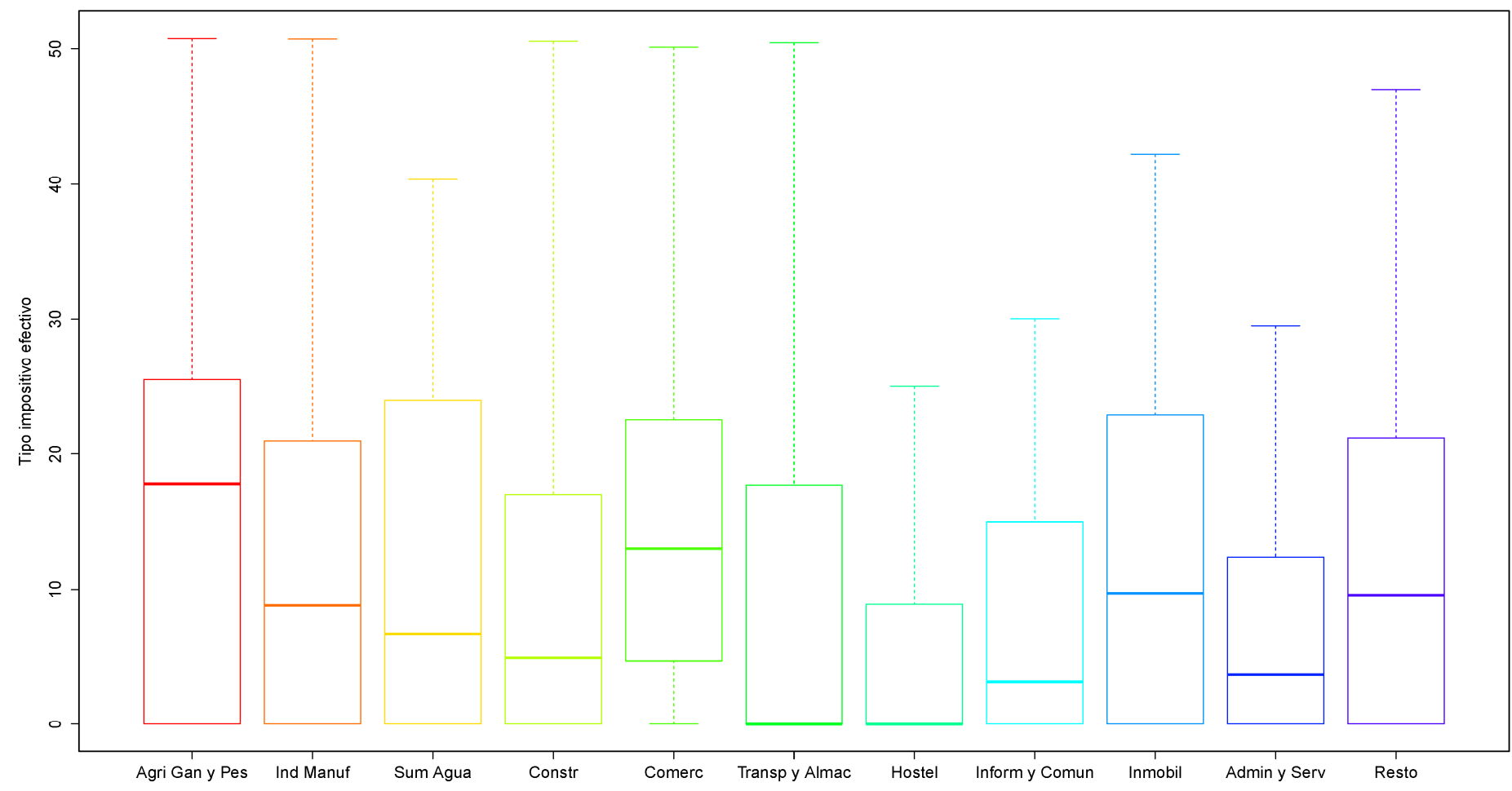


Para corroborar si las diferencias observadas en el análisis descriptivo son estadísticamente significativas hemos realizado un análisis de varianza univariante (ANOVA), donde la variable dependiente es el tipo impositivo efectivo y el factor los diferentes sectores de actividad (véase Tabla 13). Dado el nivel de significatividad de la prueba, por debajo de $1,00 \%$, se puede concluir que efectivamente el nivel impositivo efectivo difiere entre los distintos sectores de actividad, como ya se apreciaba en los resultados del análisis descriptivo.

Tabla 13. Prueba univariante del efecto del sector de actividad en el TIE

\begin{tabular}{crrc}
\hline Variable & $\begin{array}{c}\text { Medias } \\
\text { cuadradas }\end{array}$ & $\boldsymbol{F}$ & Significat. \\
\hline Sector & 1061 & 6,239 & 0,000 \\
\hline
\end{tabular}

Para finalizar el análisis hemos llevado a cabo comparaciones múltiples por parejas en los grupos que integran el factor sector de actividad para la variable dependiente tipo impositivo efectivo. De acuerdo con los resultados (véase Tabla 14) y en línea con los estadísticos descriptivos obtenidos, se aprecia que hay diferencias estadísticamente significativas entre los distintos sectores de actividad. Del análisis de los intervalos de confianza obtenidos para un nivel de significatividad del 5\% la industria manufacturera, la construcción, transporte y almacenamiento, hostelería y administración y servicios aparecen como las actividades de referencia en las comparaciones con los menores tipos impositivos efectivos. Por el contrario, los sectores con los mayores tipos impositivos efectivos son agricultura, ganadería y pesca y comercio. Sorprende que tratándose de sectores que se prestan a incluir cooperativas de las consideradas como especialmente protegidas por el RFC (agrarias y de consumidores y usuarios) su tributación efectiva sea las más elevada. Sin embargo, si tenemos en cuenta lo que se establece en el RFC (art. 15.3), estas cooperativas pueden ajustar el beneficio cooperativo a cero, por lo que su tributación estará basada sobre todo en los beneficios extracooperativos y por lo tanto al tipo general, lo que justificaría una tributación efectiva más elevada.

La importante presencia de cooperativas de la construcción en la Comunidad Valenciana hace que haya una correlación entre estas magnitudes en lo que a menor tributación efectiva se refiere. 
Tabla 14. Comparaciones múltiples por parejas del sector de actividad en el TIE

\begin{tabular}{|c|c|c|c|c|c|}
\hline & \multicolumn{4}{|c|}{ Límite intervalo } & \\
\hline Comparación & Difer. & Inferior & Superior & $P$ ajust. & \\
\hline Ind Manuf - Agri Gan y Pes & -4.54 & -7.98 & -1.10 & 0.00 & **** \\
\hline Sum Agua - Agri Gan y Pes & -4.98 & -16.43 & 6.47 & 0.95 & \\
\hline Constr - Agri Gan y Pes & -7.43 & -11.88 & -2.98 & 0.00 & $* * *$ \\
\hline Comerc - Agri Gan y Pes & -1.93 & -4.96 & 1.11 & 0.62 & \\
\hline Transp y Almac - Agri Gan y Pes & -7.85 & -13.59 & -2.12 & 0.00 & $* * *$ \\
\hline Hostel - Agri Gan y Pes & -11.04 & -22.49 & 0.41 & 0.07 & $*$ \\
\hline Inform y Comun - Agri Gan y Pes & -8.57 & -20.91 & 3.76 & 0.48 & \\
\hline Inmobil - Agri Gan y Pes & -3.71 & -9.89 & 2.47 & 0.69 & \\
\hline Admin y Serv - Agri Gan y Pes & -8.86 & -16.61 & -1.11 & 0.01 & $* * *$ \\
\hline Resto - Agri Gan y Pes & -3.56 & -9.15 & 2.03 & 0.61 & \\
\hline Sum Agua - Ind Manuf & -0.44 & -11.98 & 11.11 & 1.00 & \\
\hline Constr - Ind Manuf & -2.89 & -7.58 & 1.80 & 0.66 & \\
\hline Comerc - Ind Manuf & 2.61 & -0.76 & 5.99 & 0.31 & \\
\hline Transp y Almac - Ind Manuf & -3.31 & -9.24 & 2.61 & 0.78 & \\
\hline Hostel - Ind Manuf & -6.50 & -18.04 & 5.05 & 0.77 & \\
\hline Inform y Comun - Ind Manuf & -4.03 & -16.46 & 8.39 & 0.99 & \\
\hline Admin y Serv - Ind Manuf & -4.32 & -12.21 & 3.57 & 0.80 & \\
\hline Resto - Ind Manuf & 0.98 & -4.80 & 6.76 & 1.00 & \\
\hline Constr - Sum Agua & -2.45 & -14.34 & 9.43 & 1.00 & \\
\hline Comerc - Sum Agua & 3.05 & -8.38 & 14.48 & 1.00 & \\
\hline Transp y Almac - Sum Agua & -2.88 & -15.30 & 9.55 & 1.00 & \\
\hline Hostel - Sum Agua & -6.06 & -21.95 & 9.83 & 0.98 & \\
\hline Inform y Comun - Sum Agua & -3.60 & -20.14 & 12.94 & 1.00 & \\
\hline Inmobil - Sum Agua & 1.27 & -11.37 & 13.90 & 1.00 & \\
\hline Admin y Serv - Sum Agua & -3.88 & -17.35 & 9.59 & 1.00 & \\
\hline Resto - Sum Agua & 1.42 & -10.94 & 13.77 & 1.00 & \\
\hline Comerc - Constr & 5.50 & 1.10 & 9.90 & 0.00 & $* * *$ \\
\hline Transp y Almac - Constr & -0.42 & -6.98 & 6.14 & 1.00 & \\
\hline Hostel - Constr & -3.61 & -15.49 & 8.28 & 1.00 & \\
\hline Inform y Comun - Constr & -1.14 & -13.88 & 11.60 & 1.00 & \\
\hline Inmobil - Constr & 3.72 & -3.23 & 10.67 & 0.82 & \\
\hline Admin y Serv - Constr & -1.43 & -9.81 & 6.95 & 1.00 & \\
\hline Resto - Constr & 3.87 & -2.56 & 10.30 & 0.69 & \\
\hline Transp y Almac - Comerc & -5.93 & -11.62 & -0.23 & 0.03 & $* *$ \\
\hline Hostel - Comerc & -9.11 & -20.54 & 2.32 & 0.27 & \\
\hline Inform y Comun - Comerc & -6.65 & -18.96 & 5.67 & 0.81 & \\
\hline Inmobil - Comerc & -1.78 & -7.93 & 4.36 & 1.00 & \\
\hline Admin y Serv - Comerc & -6.93 & -14.65 & 0.79 & 0.13 & \\
\hline Resto - Comerc & -1.63 & -7.18 & 3.91 & 1.00 & \\
\hline Hostel - Transp y Almac & -3.18 & -15.61 & 9.24 & 1.00 & \\
\hline Inform y Comun - Transp y Almac & -0.72 & -13.96 & 12.52 & 1.00 & \\
\hline Inmobil - Transp y Almac & 4.14 & -3.69 & 11.98 & 0.83 & \\
\hline Admin y Serv - Transp y Almac & -1.00 & -10.13 & 8.12 & 1.00 & \\
\hline Resto - Transp y Almac & 4.29 & -3.09 & 11.67 & 0.73 & \\
\hline Inform y Comun - Hostel & 2.46 & -14.08 & 19.00 & 1.00 & \\
\hline Inmobil - Hostel & 7.33 & -5.31 & 19.96 & 0.74 & \\
\hline Admin y Serv - Hostel & 2.18 & -11.29 & 15.65 & 1.00 & \\
\hline Resto - Hostel & 7.48 & -4.88 & 19.83 & 0.68 & \\
\hline Inmobil - Inform y Comun & 4.86 & -8.58 & 18.30 & 0.99 & \\
\hline Admin y Serv - Inform y Comun & -0.28 & -14.52 & 13.95 & 1.00 & \\
\hline Resto - Inform y Comun & 5.01 & -8.17 & 18.19 & 0.98 & \\
\hline Admin y Serv - Inmobil & -5.15 & -14.56 & 4.27 & 0.80 & \\
\hline Resto - Inmobil & 0.15 & -7.58 & 7.88 & 1.00 & \\
\hline Resto - Admin y Serv & 5.30 & -3.74 & 14.33 & 0.72 & \\
\hline Inmobil - Ind Manuf & 0.83 & -5.52 & 7.18 & 1.00 & \\
\hline
\end{tabular}

NOTAS: límites del intervalo de confianza calculados para un nivel de significatividad del $5 \%$. (***) Significativo al nivel del 1,00\%

(**) Significativo al nivel del 5,00\%

(*) Significativo al nivel del 10,00\% 


\section{CONCLUSIONES}

Como consecuencia de la reforma de la legislación mercantil para adaptarse a las Normas Internacionales de Contabilidad (NIIFs), la contabilización del impuesto sobre sociedades según el método del efecto impositivo adquiere un mayor protagonismo en las cuentas anuales. Este cambio proporciona una información adicional en el balance que, junto con el gasto devengado de la cuenta de resultados, permite una estimación del impuesto pagado por las empresas mediante la aplicación de una sencilla fórmula que aplicada a los datos agregados de varios ejercicios nos ha permitido valorar y comparar la presión fiscal de las cooperativas, obteniendo resultados que a nuestro juicio nos parecen muy reveladores de las diferencias territoriales y sectoriales como consecuencia, fundamentalmente, de la convivencia de la Ley Fiscal de Cooperativas (Ley 20/1990) con una legislación autonómica diversa.

Hemos observado que el tipo impositivo efectivo según criterios fiscales para el período considerado está en torno al 15\% como valor medio de las cooperativas. Las microcooperativas son las que gozan de una menor presión fiscal (en torno al 13\%), seguidas de las grandes cooperativas con un punto porcentual superior. Las cooperativas de tamaño medio son las grandes perjudicadas con una tributación efectiva sobre el $17 \%$. Es muy probable que entre las cooperativas más pequeñas es donde más haya de las especialmente protegidas con los consiguientes beneficios fiscales adicionales. También queremos destacar que, según los resultados obtenidos y al igual que ocurre con las empresas consideradas capitalistas, las grandes cooperativas tampoco son las que tienen una tributación efectiva mayor, estando casi al nivel de las de menor tributación, probablemente debido a la aplicación mayoritaria del régimen de consolidación fiscal por formar parte de un grupo y a la realización de más inversiones en actividades que dan derecho a deducciones (I+D+i, mecenazgo, medioambientales, etc.).

Por comunidades, las cooperativas del País Vasco son las que tienen una tributación efectiva claramente inferior a las del resto $(6,5 \%)$, destacando también aunque de lejos $(11,4 \%)$ la Comunidad Valenciana. Por el contrario, las cooperativas que más han tributado han sido las de Castilla-León, Murcia, Andalucía y Galicia. Por consiguiente, las mejores condiciones fiscales de la ley foral del País Vasco estarían motivando este menor tipo impositivo, mientras que en la Comunidad Valenciana lo atribuimos sobre todo al destacado protagonismo de las de menor tamaño, además del efecto adicional en la tributación derivado 
de una mayor dotación al FRO y al FEP del los resultados extraordinarios. La falta de observaciones suficientes para la Comunidad Foral de Navarra ha impedido que nos hayamos podido pronunciar claramente sobre la fiscalidad de sus cooperativas.

Del análisis llevado a cabo por sectores, concluimos que las cooperativas agrícolas, ganaderas y pesqueras $(16,80 \%)$ junto con las dedicadas al comercio $(14,88 \%)$ son las que tienen una mayor tributación efectiva, aun cuando están entre las consideradas especialmente protegidas. Pero, teniendo en cuenta que estos sectores son precisamente los que según en el artículo 15 de la Ley 20/90 pueden dejar el resultado cooperativo a cero, su tributación podría estar basada sobre todo en sus resultados extracooperativos y, por lo tanto, sometida al tipo general. Por el contrario, la hostelería $(5,76 \%)$ se muestra como el sector más favorecido. El predominio de las cooperativas de menor tamaño entre estas últimas, con unos tipos impositivos asociados más bajos, podría ser el principal motivo de la menor carga fiscal media, además de haber contado con importantes deducciones fiscales. Pero también habría que plantear la posibilidad de que en determinados sectores de actividad sea más o menos usual fijar en sus estatutos unas aportaciones al FRO a al FEP muy superiores a los fijados en su ley reguladora, lo que podría derivar en una menor tributación efectiva.

\section{BIBLIOGRÁFÍA}

Agencia Tributaria (2011), Informe anual de recaudación tributaria 2011.

ALGUACIL MARÍ, P. y ROMERO CIVERA, A. (2013) Diferencias territoriales en el concepto de cooperativa protegida y especialmente protegida, REVESCO. Revista de Estudios Cooperativos, $\mathrm{n}^{\mathrm{o}} 110$ (1), pp. 7-42.

BUIJINK, W., JANSSEN, B. y SCHOLS, Y. (2002) Evidence of the effect of domicile on corporate average effective tax rate in the European Union, Journal of International Acconunting, Auditing \& Taxation, 11, pp. 115-130.

CALVÉ PÉREZ, J.I., LABATUT SERER, G. y MOLINA LLOPIS, R. (2005) Variables económico-financieras que inciden sobre la presión fiscal soportada por las empresas de reducida dimensión: Efectos de la reforma fiscal de 1995 en las empresas de la Comunidad Valenciana, Revista Española de Financiación y Contabilidad, 127, pp. 875897.

CHEN, S., CHEN, X., CHEN, T. y SHEVLIN, T.J. (2009) Are Family Firms More Tax Aggressive Than Non-Family Firms?, AAA 2008 Financial Accounting And Reporting 
Section (FARS) Paper, Journal of Financial Economics (JFE), Forthcoming (CAAA) 2008 Annual Conference Paper.

COLLINS, J.H. y SHACKELFORD, D.A. (1995) Corporate domicili and Average Effective Tax Rates: The Cases of Canada, Japan, The United Kingdom and the United States. Intenational Tax and Public Finance, 2, pp. 55-83.

CUBEDO TORTONDA, M. (2007) El régimen económico de las sociedades cooperativas, CIRIEC-España, Revista de Economía Publica, Social y Cooperativa, 58, pp. 161-187.

FEENY, S., GILLMAN, M. y HARRYS, M.N. (2005) Econometric Accounting of the Australian Corporate Tax Rates: A Firm Panel Example, Accounting Research Journal, 19 (1): 64-73.

FERNÁNDEZ RODRÍGUEZ, E. (2004) Los factores condicionantes de la presión fiscal empresarial española a partir de la información contable. Especial mención a las decisiones financieras", Revista Española de Financiación y Contabilidad, 120, pp. 125159.

FERNÁNDEZ RODRÍGUEZ, E. y MARTÍNEZ ARIAS, A. (2009) Factores determinantes de la presión fiscal de las empresas cotizadas en Estados Unidos y la Unión Europea a partir de la información contable, VII Workshop de Investigación Empírica en Contabilidad Financiera, Cartagena.

GARRIDO PULIDO, T. y PUENTES POYATOS, R. (2006) La presión fiscal en las sociedades cooperativas agrarias de segundo grado: repercusión de la Ley 3/2002, por la que se modifica la Ley 2/1999 de sociedades cooperativas andaluzas, REVESCO. Revista de Estudios Cooperativos, $\mathrm{n}^{\circ} 88$ (1), pp. 62-97.

GUPTA, S. y NEWBERRY, K. (1997) Determinants of the Variability in Corporate Effective Tax Rates: Evidence from Longitudinal Data, Journal of Accounting and Public Policy, 16, pp. 1-34.

KIM, K.A. y LIMPAPHAYOM, P. (1998) Taxes and Firm Size in Pacific-Basin Emerging Economies, Journal of International Accounting, Auditing \& Taxation, 7, pp. 47-68.

Ley 27/2014, de 27 de noviembre, del Impuesto sobre Sociedades.

Ley 16/2007, de 4 de julio, de reforma y adaptación de la legislación mercantil en materia contable para su armonización internacional con base en la normativa de la Unión Europea.

Ley 20/1990, de 19 de diciembre, sobre Régimen Fiscal de Cooperativas. 
MARTÍNEZ VARGAS, J. (2006) Diferencias entre contabilidad y fiscalidad en las grandes empresas españolas. Evolución de la presión fiscal en el período 1990-2002, Partida Doble, 175 , pp. 10-23.

MARTÍNEZ VARGAS, J. (2014) ¿Se puede calcular la verdadera tributación sobre beneficios en una sociedad a partir de los datos del balance y de la cuenta de resultados? Revista Impuestos, 2, pp. 59-71.

MOLINA LLOPIS, R. (2012) La presión fiscal en las cooperativas españolas durante el período 2003-2008, CIRIEC-España, Revista de Economía Publica, Social y Cooperativa, 74, pp. 39-58.

OMER, T.C., MOLLOY, K. y ZIEBART, D. (1993) An Investigation of the Firm SizeEffective Tax Rate Relation in the 1980s, Journal of Accounting Auditing and Finance, 8 (2), pp. 167-182.

Orden EHA/3360/2010, de 21 de diciembre, por la que se aprueban las normas sobre los aspectos contables de las sociedades cooperativas.

ORDÓÑEZ DE HARO, C. (2006) La fiscalidad de las sociedades cooperativas en España, CIRIEC-España, Revista de Economía Publica, Social y Cooperativa, 54, pp. 187-204.

PEÑARROCHA PINA, V. M. (2005) La adaptación sectorial del PGC a las empresas cooperativas y su repercusión fiscal, Técnica Contable, 675, pp. 16-31.

PORCANO, T.M. (1986) Corporate Tax Rates: Progressive, Proportional or Regresive, The Journal of the American Taxation Association, 7 (2), pp. 17-31.

POZUELO CAMPILlO, J., CARMONA IBÁÑEZ, P. y MARTÍNEZ VARGAS, J. (2012) Las sociedades cooperativas y las empresas capitalistas en la Comunidad Valenciana: análisis comparado de su estructura económica y financiera, CIRIEC-España, Revista de Economía Pública, Social y Cooperativa, 74, pp. 117-148.

R Development Core Team (2013) A language and environment for statistical computing, $R$ Foundation for Statistical Computing, Vienna, Austria. URL http://www.R-project.org/.

Real Decreto 1514/2007, de 16 de noviembre, por el que se aprueba el plan General de Contabilidad.

Real Decreto 1515/2007, de 16 de noviembre, por el que se aprueba el Plan General de Contabilidad de Pequeñas y Medianas Empresas y los criterios contables específicos para microempresas.

Real Decreto 2003/2009, de 23 de diciembre, por el que se modifica el Real Decreto 1514/2007, de 16 de noviembre, por el que se aprueba el Plan General de Contabilidad. 
Real Decreto legislativo 4/2004, de 5 de marzo, por el que se aprueba el Texto Refundido de la Ley del Impuesto sobre Sociedades.

REVELLE, W. (2013) psych, Procedures for Personality and Psychological Research, Northwestern University, Evanston, Illinois, USA, http://CRAN.Rproject.org/package $=$ psych Version $=1.3 .2$.

RICHARDSON, G. y LANIS, R. (2007) Determinants of the Variability in Corporate Effective Tax Rates and Tax Reform: Evidence from Australia, Journal of Accounting and Public Policy, 26, pp. 689-704.

STICKNEY, C.P. y MCGEE, V.E. (1982) Effective Corporate Tax Rates. The Effect of Size, Capital Intensity, Leverage, and Other Factors, Journal of Accounting and Public Policy, 1, pp. 125-152.

WANG, S. (1991) The Relation Between Firm Size and Effective Tax Rates: A Test of Firms` Political success, The Accounting Review, 66 (1), pp. 158-169.

ZIMMERMAN, J.L. (1983) Taxes and firm size, Journal of Accounting and Economics, 5, (2), pp. 119-149. 